\section{Taking PDE-5 data to heart}

\section{By Tim Fulmer, Senior Writer}

Phosphodiesterase-5 inhibitors like Viagra sildenafil may still have value in the cardiovascular space, even though companies focus on marketing the drugs to treat erectile dysfunction. A paper published in the Proceedings of the National Academy of Sciences now suggests that Viagra could have efficacy in preventing or at least slowing the onset of heart failure in patients with Duchenne muscular dystrophy.

Taking the mouse results in PNAS forward into humans may not be straightforward, however, as researchers polled by SciBX do not agree on the proper patient population in which to look for cardiovascular efficacy of the phosphodiesterase-5 (PDE-5) inhibitors.

In cardiac muscle, inhibiting PDE-5 directly increases levels of the signaling molecule cyclic guanosine monophosphate (cGMP), which leads to multiple downstream effects that could benefit a failing heart. These include relaxation of cardiac muscle and downregulation of genes that contribute to aberrant muscle remodeling under cardiac stress (see Figure 1, "Rescuing a failing heart"). ${ }^{1}$ Evidence from multiple preclinical cardiomyopathy models suggests that cGMP protein kinase-1 (PKG-1) helps mediate these effects. ${ }^{2}$

The PNAS paper looked at the $m d x$ mouse, which is a model for dystrophin-deficient forms of cardiomyopathy that occur in Duchenne muscular dystrophy (DMD). Dystrophin is a cytoskeletal protein that provides structural support to the muscle cell and links the contractile apparatus to the extracellular matrix.

Previously, the same researchers at the University of Montreal and McGill University found that $m d x$ mice had altered cardiac energy metabolism, which suggested that defective cGMP signaling may occur prior to overt cardiomyopathy. ${ }^{3}$

To test the hypothesis that enhancing cGMP signaling could help prevent cardiomyopathy in the same mouse model, the Canadian researchers explored genetic and pharmacological strategies to boost early activation of the cGMP pathway. ${ }^{4}$

In one set of experiments, $m d x$ mice were engineered to overexpress a constitutively active form of guanylyl cyclase (GC) in cardiomyocytes. GC catalyzes the conversion of GMP to cGMP, which activates PKG-1 and induces downstream muscle relaxation, among other effects (see Figure 1, "Rescuing a failing heart").

Working hearts isolated from 20 -week-old $m d x$ mice overexpressing the GC transgene showed significantly better contractile performance and membrane integrity than working hearts isolated from $m d x$ mice that lacked the transgene $(p<0.05)$.
In a second set of experiments, $m d x$ mice were treated with sildenafil. Researchers found that the compound significantly improved heart rate in response to stress and significantly minimized cardiomyocyte injury compared with placebo ( $p<0.01$ and $p<0.05$, respectively).

The authors concluded that their study "raises the possibility that a new therapeutic approach based on cGMP up-regulation with PDE-5 inhibitors" could help treat or protect against cardiomyopathy associated with dystrophin deficiency.

"The PNAS study shows that defective cGMP signaling may play a critical role in the pathogenesis of preclinical-stage Duchenne cardiomyopathy," said Dongsheng Duan, associate professor of molecular microbiology and immunology at the University of Missouri.

Duan and colleagues have shown that dystrophin gene therapy can prevent development of cardiomyopathy in aged $m d x$ mice. ${ }^{5}$ Although that finding helped illustrate how mechanical defects might impact muscle pathology in DMD patients, Duan noted the PNAS paper suggests signaling defects also could be at work.

But Elmar Burchardt, VP of medical affairs at Aastrom Biosciences Inc., said it was unexpected that a defect in the structural protein dystrophin could be at least partially offset by "a seemingly mechanistically unrelated therapy" that targets cGMP intracellular signaling.

"It would be interesting to study gene expression profiles to elucidate potential mechanisms," he said.

Aastrom's Cardiac Repair Cells, an autologous mixture of stem and early progenitor cells delivered to the heart muscle, is in Phase I testing to treat dilated cardiomyopathy.

\section{Into the clinic}

Although the mechanism is being sorted out, academics are taking sildenafil and other PDE-5 inhibitors into human clinical trials in the cardiovascular space. Up for debate is the specific indication in which such compounds should be tested.

Some researchers are taking an all-comers approach and are testing PDE-5 inhibitors in broad heart-failure populations. Others, however, think the compounds may be better suited to treating cardiomyopathy in patients with DMD.

David Kass, professor of cardiology and biomedical engineering at the Johns Hopkins School of Medicine, and Margaret Redfield, professor of medicine in the Department of Cardiovascular Diseases at the Mayo Clinic, are in the former camp. Kass told SciBX that he and colleagues are running a trial of Cialis tadalafil "to treat or prevent ventricular hypertrophy in patients on antihypertensives. The primary endpoint, reduction in cardiac mass, is being monitored with MRI."

Cialis, a PDE-5 inhibitor from Eli Lilly and Co., is marketed to treat erectile dysfunction (ED).

Redfield is a principal investigator for a similar trial organized by the Heart Failure Clinical Research Network that is expected to start in 2H08. The Phosphodiesterase-5 Inhibition to Improve Clinical Status and Exercise Capacity in Diastolic Heart Failure (RELAX) trial "will test the hypothesis that chronic PDE-5 inhibition will improve exercise performance and clinical outcomes in diastolic heart failure," 
she said. "The impact of therapy on several physiological endpoints will be tested, including humoral function, renal function, LV [left ventricular] mass and diastolic function, and aortic stiffness."

Patients in RELAX will receive sildenafil or placebo.

Elizabeth McNally, professor of medicine and director of the Institute of Cardiovascular Research at the University of Chicago, suggested a different tack-studying PDE-5 inhibitors in DMD patients who have cardiomyopathy. She did, however, caution that "this could be risky, since later-stage DMD patients are typically hypotensive and have accompanying arrhythmias," she said.

Indeed, the current label for Viagra states that some patients with "preexisting cardiovascular risk factors" have reported ventricular arrhythmia, among other cardiovascular events, when using the drug.

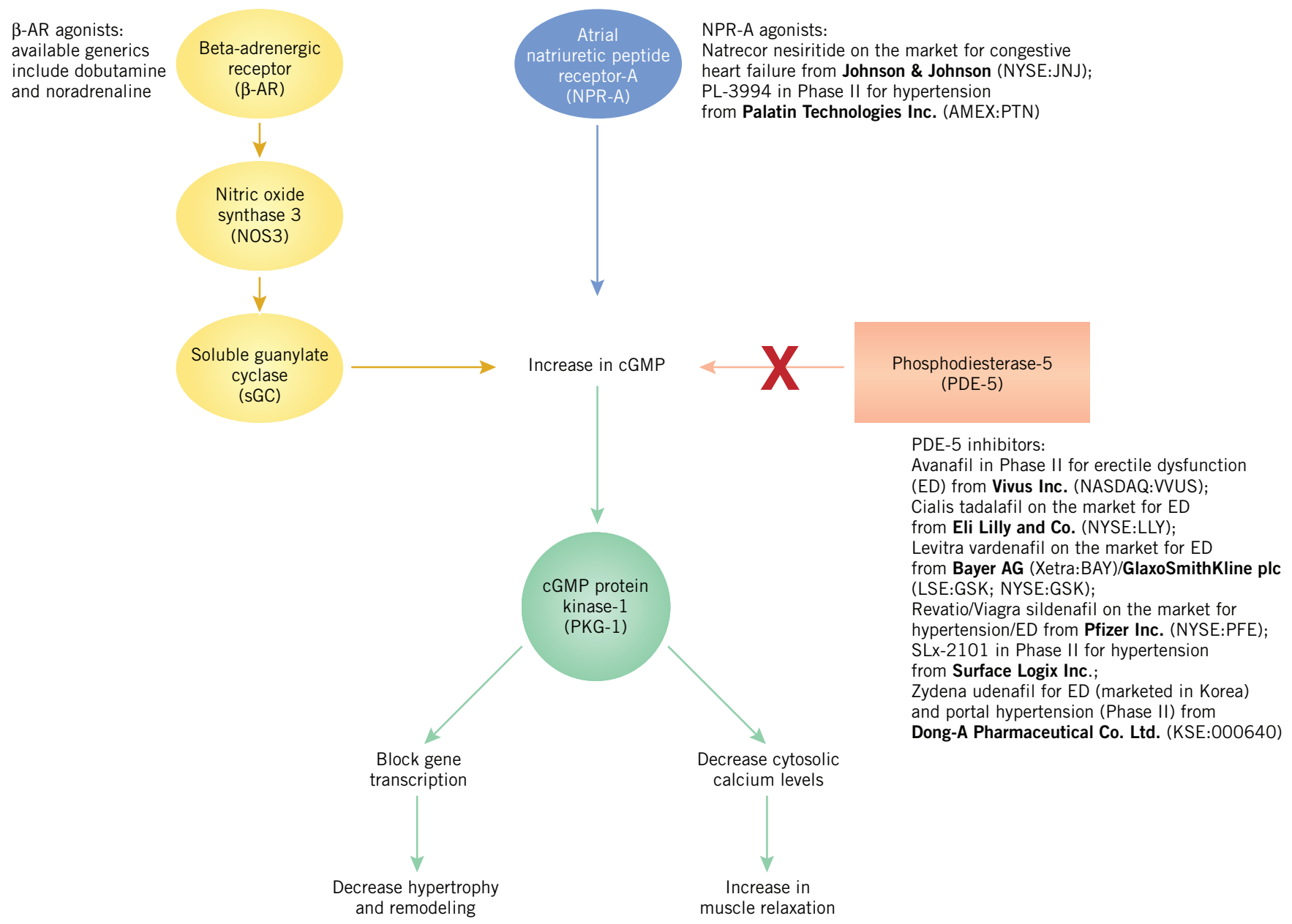

Figure 1. Rescuing a failing heart. In response to a decline in pumping capacity, heart muscle activates a number of mechanisms to initiate cardiac repair. However, when these mechanisms are active over long periods of time, they result in significant cardiac remodeling and hypertrophy, which can progress to cardiomyopathy and heart failure.

Work published last month in PNAS by Khairallah et al. and research by others suggest that increasing levels of cyclic guanosine monophosphate (cGMP) in the cardiomyocyte not only helps slow or prevent cardiac remodeling and hypertrophy but also improves heart contractility.

In dystrophin-deficient mice, researchers showed that increasing levels of cGMP by inhibition of phosophodiesterase-5 (PDE-5) improved cardiomyocyte integrity and contractile performance. Other researchers have shown that the downstream cardioprotective effects of increased cGMP levels are at least partly mediated by cGMP protein kinase-1 (PKG-1). ${ }^{1,2}$

The figure illustrates two other strategies for increasing cGMP in the cardiomyocyte that have been explored. Both use agonists of surface receptors $-\beta$-adrenergic receptor $(\beta-A R)$ and atrial natriuretic peptide receptor-A (NPR-A) - to activate intracellular signaling pathways that enhance downstream levels of cGMP. It should be noted that $\beta$-AR can activate a cyclic adenosine monophosphate (cAMP) pathway, which leads to muscle contraction, in addition to the cGMP pathway illustrated that causes muscle relaxation.

The figure shows selected compounds in development or on the market that enhance intracellular levels of cGMP. Nitric oxide synthase 3 (NOS3) or soluble guanylate cyclase (sGC) also could be potentially targeted to increase downstream cGMP. 
Jeffrey Towbin, professor and chief of pediatric cardiology at the Texas Children's Hospital, proposed a middle ground. He said that next steps could include investigating PDE-5 inhibition in patients with "all-cause forms of dilated cardiomyopathy." Dilated cardiomyopathy is the most common form of cardiomyopathy, and dystrophin is usually disrupted in end-stage forms of the disease, Towbin said.

Carl Spana, CEO of Palatin Technologies Inc., wanted to see "how early in the disease process sildenafil treatment needs to be started to achieve maximal benefit, and how late in disease intervention can be started and still have benefit."

It would also be useful to determine "the effects-both positive and negative- of chronic long-term treatment of dystrophic cardiomyopathy with sildenafil," he added.

Palatin's PL-3994, a subcutaneously delivered atrial natriuretic peptide receptor-A (NPR-A) agonist, is in Phase IIa testing to treat hypertension. The compound is expected to enter a Phase II trial this year to treat acute decompensated congestive heart failure, which will evaluate reduction and maintenance of pulmonary capillary wedge pressure and diuresis, Spana said.

According to Christine Des Rosiers, corresponding author on the PNAS paper, "in the longer term, we hope to find collaborators to help design and organize a multicenter trial that might investigate PDE5 inhibitors as part of combination therapy to treat heart failure in muscular dystrophy patients." Des Rosiers is a professor in the Department of Nutrition at the University of Montreal and a researcher at the Montreal Heart Institute.

Indeed, regardless of indication, the realities of treating heart failure will likely require that PDE-5 inhibitors be used in combination with a variety of other drugs.

"At any given time, heart failure patients might take anywhere from 5-10 medications to alleviate heart failure symptoms," Des Rosiers noted. "Clearly, then, we have to consider PDE-5 inhibition for heart failure in muscular dystrophy patients in the context of a combination therapy-which could mean running trials that combine PDE-5 inhibitors with, for example, an approved ACE [angiotensin-converting enzyme] inhibitor."

\section{Alternatives to PDE-5 inhibition}

Some companies in the heart failure space believe there are better disease-modifying strategies than PDE-5 inhibition.

"Agents such as $\beta$-adrenergic receptor agonists and phosphodiesterase inhibitors work to increase cardiac systolic function indirectly, by first increasing intracellular calcium levels, which subsequently activate myosin. However, increasing calcium levels also has the potential for causing arrhythmia, as well as inducing ischemia from increased myocardial oxygen consumption," said Andrew Wolff, CMO and VP of clinical R\&D at Cytokinetics Inc.
"To avoid those risks, Cytokinetics has adopted the strategy of improving systolic function by direct activation of myosin," he said. "CK-1827452, our small molecule myosin activator, accelerates the rate-limiting step of the actin-myosin enzymatic cycle without altering calcium levels.”

In animal models of heart failure, as well as in a Phase I trial in healthy volunteers, the compound improved cardiac pumping function without increasing the velocity of cardiac contraction, which can lead to excessive oxygen consumption.

CK-1827452 is in two placebo-controlled Phase IIa trials in patients with stable heart failure.

Miragen Therapeutics Inc. is targeting microRNAs in heart disease rather than individual enzymes like PDE-5. Eric Olson, company cofounder and professor of molecular biology at the University of Texas Southwestern Medical Center, told SciBX that Miragen prefers its strategy because "targeting an individual microRNA can potentially modulate multiple components of a cellular pathway implicated in heart disease."

Olson and colleagues identified signature patterns of miRNAs that are dysregulated in cardiac hypertrophy and heart failure in humans and mice. ${ }^{6}$ In mice subjected to cardiac stress, knockout of cardiac-specific miRNA-208 led to decreased hypertrophy, fibrosis and $\beta$-myosin heavy chain expression compared with what was seen in wild-type animals.?

Miragen is now studying synthetic anti-miRNA therapeutics in rodent models of heart disease and plans also to look at efficacy and safety in sheep and pigs before humans, President and CEO William Marshall told SciBX.

\section{REFERENCES}

1. Heineke, J. \& Molkentin, J. Nat. Rev. Mol. Cell Biol. 7, 589-600 (2006)

2. Kass, D. et al. Circ. Res. 101, 1084-1095 (2007)

3. Khairallah, M. et al. J. Mol. Cell. Cardiol. 43, 119-129 (2007)

4. Khairallah, M. et al. Proc. Natl. Acad. Sci. USA; published online May 13, 2008; doi:10.1073/pnas.0710595105

Contact: Christine Des Rosiers, University of Montreal, Montreal, Quebec, Canada

e-mail: christine.des.rosiers@umontreal.ca

5. Bostick, B. et al. Circ. Res. 102, 121-130 (2008)

6. van Rooj, E. et al. Proc. Natl. Acad. Sci. USA 103, 18255-18260 (2006)

7. van Rooj, E. et al. Science 316, 575-579 (2007)

\section{COMPANIES AND INSTITUTIONS MENTIONED}

Aastrom Biosciences Inc. (NASDAQ:ASTM), Ann Arbor, Mich. Cytokinetics Inc. (NASDAQ:CYTK), South San Francisco, Calif.

Eli Lilly and Co. (NYSE:LLY), Indianapolis, Ind.

Johns Hopkins School of Medicine, Baltimore, Md.

Mayo Clinic, Rochester, Minn.

McGill University, Montreal, Quebec, Canada

Miragen Therapeutics Inc., Boulder, Colo.

Montreal Heart Institute, Montreal, Quebec, Canada

Palatin Technologies Inc. (AMEX: PTN), Princeton, N.J.

Texas Children's Hospital, Houston, Texas

University of Chicago, Chicago, III.

University of Missouri, Columbia, Mo.

University of Montreal, Montreal, Quebec, Canada

University of Texas Southwestern Medical Center, Dallas, Texas 